\title{
GENERALIZACIÓN FRACTAL DE CÉLULAS PRENEOPLÁSICAS Y CANCERÍGENAS DEL EPITELIO ESCAMOSO CERVICAL. UNA NUEVA METODOLOGÍA DE APLICACIÓN CLÍNICA
}

\author{
Javier Rodríguez V. MD. ${ }^{1 *}$, Signed Prieto ${ }^{2}$, Catalina Correa ${ }^{2,3}$, Héctor Posso MD. M. Sc. ${ }^{4}$, \\ Pedro Bernal ${ }^{2}$, Germán Puerta 5 , Sarith Viterr ${ }^{6}$, INGRId Rojas ${ }^{6}$. \\ ${ }^{1}$ Director del Grupo Insight. Director de la Línea de Profundización e Internado Especial: Física y Matemáticas \\ Aplicadas a la Medicina, Universidad Militar Nueva Granada. ${ }^{2}$ Investigadora Grupo Insight. ${ }^{3}$ Profesora de \\ la Línea de Profundización e Internado Especial: Física y Matemáticas Aplicadas a la Medicina, Universidad \\ Militar Nueva Granada. ${ }^{4}$ Docente Universidad Militar Nueva Granada. ${ }^{5}$ Internado especial: Física y \\ Matemáticas Aplicadas a la Medicina, Universidad Militar Nueva Granada. ${ }^{6}$ Línea de profundización: Física y \\ Matemáticas Aplicadas a la Medicina, Universidad Militar Nueva Granada.
}

\section{Resumen}

\begin{abstract}
A partir de la evaluación de las dimensiones fractales y aplicando el concepto de variabilidad y armonía matemática intrínseca celular (AMI), se desarrolló un método matemático de aplicación clínica para el diagnóstico de células preneoplásicas y neoplásicas del epitelio escamoso cervical, el cual supera el diagnóstico de ASCUS (células escamosas atípicas de significado no determinado). A partir de un método desarrollado previamente para el diagnóstico de células normales, ASCUS y L-SIL, se realizaron permutaciones estructurales fractales con los valores generales de la AMI y de variabilidad fractal para normalidad y enfermedad, buscando los prototipos generales de células normales, preneoplásicas y neoplásicas. Se midieron cinco células ASCUS y cinco cancerígenas del epitelio escamoso del cuello uterino y se encontró que para la normalidad hay 18 prototipos, mientras que para la anormalidad 44, incluyendo todos los estados de evolución hasta carcinoma. Estos resultados fueron confirmados al comparar los resultados obtenidos por las técnicas convencionales con los obtenidos por esta metodología; lo que hace pensar que se desarrolló una nueva metodología que permite diferenciar los distintos tipos de células del epitelio escamoso del cuello uterino (normales o cancerígenas).
\end{abstract}

Palabras clave: cáncer de cuello uterino, fractales, citología

\section{FRACTAL GENERALIZATION OF PRE-NEOPLASIC CERVICAL EPITHELIAL CELLS FOR CLINICAL APPLICATION}

\begin{abstract}
Beginning with the evaluation of fractal dimensions, and applying the concept of variability and Cellular Intrinsic Methematical Harmony, a mathematical method for clinical application was developed, about the diagnosis of preneoplastic and neoplastic squamous epithelial cervix cells, which improves the ASCUS (Atypical Squamous Cells of Undetermined Significance) diagnosis. Beginning with a method previously developed for the diagnosis of normal, ASCUS and L-SIL cells, fractal structural permutations with the general Intrinsic Mathematical Harmony (IMH) and variability values for normality and disease were made, looking for the general prototypes of normal cells, premalignant and carcinogen. 5 ASCUS and 5 carcinogen cells of the squamous epithelial cervix were measured. It was found that there are 18 normal prototypes, while there were obtained 44 prototypes for the abnormality, including all stages of evolution to carcinoma. These results were confirmed by comparing the obtained results by the conventional techniques
\end{abstract}

* Correspondencia: Javier Rodríguez Velásquez grupoinsight2025@yahoo.es. Dirección Postal: Tr. 3 No 49-00. Facultad de Medicina Universidad Militar Nueva Granada, Bogotá D.C., Colombia. Teléfono: 4527541

Recibido: Septiembre 8 de 2010 Aceptado: Diciembre 15 de 2010 
with the results obtained by this methodology; making thinking that it was developed a new methodology that allows to differentiate the distinct types of the squamous epithelial cervix cells (normal or carcinogen).

Key words: uterine cervical neoplasms, fractals, cytology

\title{
GENERALIZAÇÃO FRACTAL DE CÉLULA PRÉ-NEOPLÁSICAS E CANCER DO EPITÉLIO ESCAMOSO CERVICAL. UMA NOVA METODOLOGIA DE APLICAÇÃO CLÍNICA
}

\begin{abstract}
Resumo
A partir da avaliação da dimensão fractal e aplicação do conceito de variabilidade e harmonia matemática subjacente celular (IAM), desenvolveu-se um método matemático para a aplicação clínica o diagnóstico de células pré-neoplásicas e neoplásicas do epitélio escamoso cervical, o que excede o diagnóstico ASCUS (células escamosas atípicas de significado indeterminado). Baseado em um método desenvolvido anteriormente para o diagnóstico de células normais, ASCUS e LSIL, foram realizadas permutações estruturais fractais com os valores gerais da AMI e variabilidade fractal para o normal e doença, procurando protótipos gerais de células normais, pré-neoplásicas e neoplásicas. Foram medidos cinco células ASCUS e cinco de câncer de epitélio escamoso cervical e encontrou-se que existem para o normal 18 protótipos, enquanto que para a alteração 44, incluindo todas as fases de evolução para carcinoma. Estes resultados foram confirmados através da comparação dos resultados obtidos por técnicas convencionais com aqueles obtidos por este modelo matemático, sugerindo que desenvolveu-se uma nova metodologia para diferenciar diferentes tipos de células do epitélio escamoso cervical (normais ou cancerosos).
\end{abstract}

Palavras-chave: neoplasias do colo do útero, fractais, citologia

\section{Introducción}

El sistema de Bethesda es un sistema de clasificación de grados de lesión o alteraciones celulares preneoplásicas de cuello uterino (1); en este sistema, la evaluación de las alteraciones celulares está basada en la observación cualitativa de características específicas, hecho que puede llevar a presentar una variabilidad en la diferenciación de anormalidades en lesiones del mismo grado, y que pueden generar problemas en la precisión del diagnóstico (2). Dentro de este sistema, se unificaron criterios y se introdujo la categoría celular, dando origen a una clasificación denominada ASCUS, en la cual se reúnen diferentes tipos de células que presentan anormalidades no concluyentes pero potencialmente graves o "zona gris" entre cambios celulares y lesión intraepitelial (2).

Previamente Rodríguez y cols. habían desarrollado una evaluación diagnóstica fractal a partir de la definición de los conceptos de AMI y variabilidad (en proceso de publicación), para evaluar las relaciones entre las dimensiones fractales calculadas con el método de Box-counting (Ecuación 1), de tres objetos definidos en la célula: núcleo $(\mathrm{N})$, citoplasma $(\mathrm{N})$ y célula $(\mathrm{T})$.
En dicho trabajo se tomaron ocho células del epitelio escamoso cervical, de las cuales cuatro habían sido clasificadas por los parámetros clínicos establecidos como normales, dos como ASCUS y dos como LSIL. Al comparar las dimensiones fractales de los tres objetos por parejas, se encontraron rangos de valores de AMI y variabilidad que diferencian células normales de las clasificadas como L-SIL, que permitían evaluar cuantitativamente las células clasificadas como ASCUS (células indeterminadas evaluadas subjetivamente), mostrando que una de estas células presentaba valores de AMI y variabilidad de normalidad, mientras que la otra presentaba parámetros de anormalidad.

Esta metodología constituyó la primera evaluación objetiva y cuantitativa geométrica general de diagnóstico preventivo para lesiones cervicales preneoplásicas, que por su carácter físico y matemático, es independiente de análisis estadísticos o poblacionales y de variables como la edad, factores de riesgo, etc. Al evidenciar cuantitativamente relaciones fractales, se hace innecesario el uso de las clasificaciones descriptivas utilizadas actualmente para alteraciones preneoplásicas, solucionando la dificultad diagnóstica de las células ASCUS. 
En el trabajo ya mencionado, los valores de AMI y variabilidad de las células con L-SIL se encontraron entre 0 y 1 al comparar $\mathrm{C}$ y $\mathrm{T}$, y entre 1 y 2 al comparar $\mathrm{N}$ y $\mathrm{T}$, mientras que al comparar $\mathrm{C}$ y $\mathrm{N}$ se encontró una variabilidad de 2 . Para las células normales, tanto las comparaciones entre $\mathrm{C}$ y $\mathrm{N}$ como entre $\mathrm{N}$ y $\mathrm{T}$ los valores fueron entre 4 y 5 , y la comparación entre $C$ y $\mathrm{T}$ siempre presentó un valor de 0 . Estas medidas evidenciaron que la disminución en las comparaciones entre $\mathrm{N}$ y $\mathrm{C}$ y $\mathrm{N}$ y $\mathrm{T}$ puede estar relacionada con alteraciones preneoplásicas de gravedad progresiva.

El concepto de AMI y de variabilidad de la dimensión fractal es desarrollado a partir de dos conceptos utilizados en otros trabajos: la variabilidad, desarrollada para evaluar la dinámica de la ramificación coronaria izquierda $(3,4)$, y la AMI arterial que reveló un orden geométrico subyacente de las capas arteriales (5). El trabajo que se presenta en este artículo es un estudio teórico físico matemático aplicado a la medicina, que tiene como objetivo confirmar y generalizar el diagnóstico desarrollado previamente con ocho prototipos celulares, cuatro normales, dos L-SIL y dos ASCUS, obteniendo la totalidad de prototipos de normalidad y anormalidad, que incluyen todas las posibles células que se puedan encontrar en el cuello uterino.

\section{Antecedentes}

En general, las formas que se presentan en la naturaleza son irregulares (6) y para medirlas se aproximan a objetos de la geometría euclidiana; sin embargo, la confianza en la utilización de esta geometría para medir este tipo de formas se vio alterada a finales del siglo XX, cuando se mostró que al aplicarla sobre la costa de Gran Bretaña, conducía a resultados no concluyentes, evidenciando así la necesidad de usar una nueva geometría, que fuese más adecuada para la medición de los objetos naturales (6,7). La geometría fractal fue desarrollada para el estudio de los objetos naturales irregulares, estableciéndose así una nueva forma de medida adecuada para estudiarlos. En esta geometría se utiliza la dimensión fractal, una medida numérica adimensional que caracteriza la irregularidad de los objetos, y el método más usado para medir la irregularidad de fractales salvajes o los objetos de la naturaleza es el de Box-Counting (8).

Esta geometría se ha aplicado en el estudio de diferentes dinámicas así como a estructuras anatómicas, permitiendo establecer diferencias morfológicas y fisiológicas asociadas a estados de enfermedad y normalidad (916), específicamente en cáncer (17-25). La aplicación de esta geometría a diferentes estructuras anatómicas, tanto a nivel experimental como clínico, ha permitido obtener caracterizaciones objetivas que diferencian normalidad de enfermedad, en áreas en las que la aplicación de la geometría euclidiana no había podido producir respuestas objetivas. Un ejemplo de ello son los trabajos realizados por Gazit y cols. (20), quienes diferenciaron con dimensiones fractales, la vasculatura tumoral en tejido ocular de ratones de una normal, tanto a nivel morfológico como fisiológico, logrando asî diferenciar tejidos normales de los anormales encontrados en los tumores andrógeno dependientes (21).

Polhman y cols. (22) y Lefebvre y Benali (23) encontraron que la aplicación de geometría fractal puede ayudar a disminuir el número de falsos positivos encontrados en la evaluación de mamografías digitales; Landini y Rippin (24), realizaron un análisis multifractal del tejido conectivo de interfase de la mucosa oral con diferentes grados de lesión neoplásica, y encontraron un espectro de valores que permitían la discriminación de los diferentes grados de lesión. Luzi et al. (25), observaron que las dimensiones fractales de muestras de cáncer invasivo de vejiga de alto grado, presentaban valores mayores que las de bajo grado. Sin embargo, se ha encontrado que la observación de dimensiones fractales aisladas puede ser insuficiente para establecer evaluaciones de aplicación experimental efectiva.

Rodríguez y cols. (26) mostraron que la medición de dimensiones fractales de células del epitelio escamoso de cuello uterino era insuficiente para establecer diferencias cuantitativas entre células provenientes de pacientes con diferentes diagnósticos. Este mismo grupo (5), evaluó la eficacia del octreótido de la somatostatina para inhibir el proceso de reestenosis en arterias de porcinos, desarrollando una nueva metodología de evaluación, basada en el concepto de AMI entre las dimensiones fractales de las partes de la arteria con su totalidad, y diferenciando matemáticamente normalidad de enfermedad. Continuando con estas investigaciones, se desarrolló la primera generalización matemática que determina la totalidad de posibles prototipos arteriales, desde normal hasta la oclusión total de la luz, con base en medidas de dimensión fractal y el concepto de AMI (27). De este modo se determinó un número finito de prototipos arteriales, en donde 200 correspondientes a normal y 69.049 a enfermedad, para un total de 69.249, haciendo innecesario el uso de clasificaciones descripti- 
vas de grados de estenosis o restenosis. Este resultado fue corroborado con las medidas obtenidas empíricamente, al encontrarse incluidas dentro de los prototipos.

Desde esta línea de trabajo Rodríguez y col. han desarrollado diversos métodos de caracterización y diagnóstico matemático con base en geometría fractal, para analizar diferentes exámenes como arteriografías coronarias $(3,4)$, ventriculografía izquierda (28) y ecocardiografías pediátricas (29). En la monitoría fetal desarrollaron un diagnóstico matemático basado en una reinterpretación de la concepción salud-enfermedad de los sistemas dinámicos a partir de la dimensión fractal de Zipf-Mandelbrot, que simplifica la evaluación de la monitoría estableciendo medidas objetivas y reproducibles, independientes de la experiencia del observador (30) y que fue posteriormente mejorado y refinado (31). En el campo de la morfología y función celular, se desarrolló un método de evaluación del estado eritrocitario, que permite determinar objetivamente la viabilidad de los eritrocitos en las bolsas utilizadas para transfusiones (32).

Los trabajos basados en teorías o generalizaciones físicas y matemáticas como los sistemas dinámicos, la geometría fractal y la probabilidad o la entropía, han permitido obtener métodos diagnósticos $(5,27-31)$ de aplicación experimental y clínica y métodos predictivos en inmunología, biología molecular y epidemiología. En el campo de la biología molecular y de la inmunología se han desarrollado múltiples caracterizaciones y predicciones (33-39), tales como la teoría predictiva de péptidos antigénicos presentados por el HLA clase II (40). A partir de las probabilidades de agrupación de aminoácidos en secuencias de péptidos de cuatro proteínas de superficie del merozoíto, se desarrolló una metodología para la predicción de péptidos de malaria de alta unión y de no unión, implicados en la invasión al glóbulo rojo, con una sensibilidad del 95\% y una especificidad del 90\% (41).

En cardiología, se desarrolló una evaluación de atractores cardiacos con base en su ocupación en el espacio fractal de Box-counting, que diferencia enfermedad crónica y normalidad de enfermedad aguda (42), así como una metodología de aplicación clínica que diferencia normalidad de enfermedad, basada en la probabilidad aplicada a rangos de aparición de la frecuencia cardiaca y número de latidos (43). Posteriormente a partir de frecuencias cardiacas registradas en los holters, se construyeron atractores que fueron analizados con las leyes de probabilidad y proporciones de la entropía, desarrollando un nuevo método de ayuda diagnóstica de aplicación clínica (44). En el campo de la epidemiología, basados en el cálculo de probabilidades y en la definición de rangos de números de infectados se caracterizó anualmente la dinámica temporal de malaria en Colombia (45). Así mismo se desarrolló una predicción de la dinámica de dengue en Colombia con base en la caminata al azar probabilista (46).

También se desarrolló una metodología que a partir de medidas de probabilidad y entropía, predice espacio temporalmente la aparición de brotes de malaria en 820 municipios colombianos en tres semanas, superando las predicciones temporales basadas en canales epidemiológicos que, cuando pueden lograrse, requieren información previa de cinco a siete años (47).

\section{Materiales y Métodos}

Ecuación 1: Dimensión fractal de Box-Counting:,

$$
D=\frac{\log N\left(2^{-(K+1)}\right)-\log N\left(2^{-K}\right)}{\log 2^{k+1}-\log 2^{k}}=\log _{2} \frac{N\left(2^{-(k+1)}\right)}{N\left(2^{-k}\right)}
$$

donde: $\mathbf{N}$ : número de cuadros que contiene el contorno del objeto, $\mathbf{K}$ : grado de partición de la cuadrícula y $\mathbf{D}$ : dimensión fractal.

A partir de las medidas de las dimensiones fractales y de AMI celular y variabilidad, obtenidas en el diagnóstico desarrollado previamente, se realizaron todas las permutaciones de las tres comparaciones obtenidas: $\mathrm{C}$ y N, Ny T y C y T. Para ello, se tomaron los rangos de valores que pueden tomar la AMI celular y variabilidad para normalidad y anormalidad y a partir de estos valores, se realizaron las permutaciones matemáticas para hallar los prototipos de normalidad y anormalidad. Posteriormente se analizaron los prototipos hallados para células diagnosticadas como indeterminadas o ASC-US, comparándolos con los demás prototipos, en busca de posibles diferencias o similitudes.

Posteriormente se aplicó esta metodología a cinco células provenientes de citologías diagnosticadas como ASCUS y cinco diagnosticadas como cancerígenas por un especialista, de acuerdo con los parámetros convencionales. Las muestras fueron tomadas del Laboratorio de Patología de la Liga contra el Cáncer, Seccional Bogotá, y la digitalización se hizo en el Laboratorio de Investigaciones de la Facultad de Medicina de la Universidad Militar Nueva Granada, en donde se determinó el prototipo de cada una de ellas. Por tratarse de una generalización matemática basada en la geometría fractal no es necesario tener en cuenta métodos estadísticos. 
Este trabajo se hizo de acuerdo con las guías éticas del artículo 11 de la resolución 008430 de 1993, del Ministerio de salud de Colombia, donde está clasificado en la categoría de investigación sin riesgo, pues lo que se hacen son cálculos matemáticos sobre resultados de exámenes de la práctica clínica que han sido prescritos médicamente, protegiendo la integridad y anonimato de los participantes. Los cálculos y resultados no afectaron a los pacientes, porque no fueron tomados en cuenta para el manejo clínico del paciente, ni tampoco se les suspendió el tratamiento.

\section{Limitaciones}

Para la aplicación de esta metodología es necesario tener en cuenta varios factores inherentes a la técnica de la citología. Por ejemplo, la técnica colorimétrica utilizada, la presencia de histocitos o los procesos de desecación y glicogenización que pueden modificar la relación núcleo/citoplasma, asemejando sus relaciones matemáticas a las de una célula ASCUS. Además, estas proporciones también pueden verse afectadas por los ciclos hormonales naturales, y las células perimenopáusicas pueden confundirse con células intermedias.

\section{Resultados}

Se encontró que la totalidad de prototipos para las células de cuello uterino (células normales, estadios preneoplásicos y células cancerigenas) fue 62 , de los cuales 18 eran el número de prototipos para la normalidad, mientras que para la anormalidad eran 44 , entre ellos ocho para carcinoma. En la tabla 1 se muestran 50 prototipos de todos los estados, incluyendo los resultados de las cinco células ASCUS y las cinco cancerígenas medidas (en gris).

Al analizar las permutaciones halladas para las células ASCUS se estableció que no presentan prototipos particulares sino que sus relaciones presentan valores que se obtienen para cualquier prototipo, tanto normal como anormal; de esta forma se explica matemáticamente la razón por la que las células ASCUS presentan dificultades diagnósticas (tabla 1). Adicionalmente se encontró, que las células cancerígenas medidas empíricamente presentan valores matemáticos correspondientes a prototipos de células anormales, específicamente con valores de 0 en las tres relaciones de variabilidad y AMI (tabla 1 ).
Cuando se hacen las comparaciones de las medidas obtenidas tanto para los prototipos matemáticos de $\mathrm{N}$ y $\mathrm{C}$ y N y $\mathrm{T}$ como de las evaluadas empíricamente, se puede observar que hay una asociación entre el aumento del grado de anormalidad y la disminución de los valores de variabilidad y AMI.

\section{Discusión}

En este trabajo se desarrolló una nueva metodología de aplicación clínica, en la que se cuantifican matemáticamente las relaciones fractales preneoplásicas y neoplásicas de las células del epitelio escamoso cervical, a partir de la evaluación de la variabilidad y la AMI celular de las dimensiones fractales de tres objetos: el N, el C y la T. Generalizando la evaluación desarrollada previamente con desarrollos computacionales de permutaciones estructurales fractales, se hallaron todos los prototipos geométricos celulares que representan a todas las posibles células que se pueden presentar en las citologías, proporcionando una evaluación objetiva y reproducible de normalidad, enfermedad y evolución entre las dos, corroborada empíricamente con células ASCUS y cancerígenas. Tanto los resultados teóricos como los empíricos revelan una relación entre alteraciones preneoplásicas y la disminución de los valores de variabilidad y AMI, cuando se hacen las comparaciones entre $\mathrm{N}$ y citoplasma y entre $\mathrm{N}$ y $\mathrm{T}$.

La evaluación de la citología cérvico vaginal se hace a partir de la observación cualitativa de características específicas, que son la base de los diferentes sistemas de clasificación del grado de lesión (2). Una de las nomenclaturas mas usadas para la evaluación de la citología es el sistema Bethesda, que incluye una denominación especial para cambios celulares no conclusivos pero potencialmente graves, las células escamosas atípicas de significado indeterminado o ASCUS, con el fin de acotar con más precisión la "zona gris" entre cambios celulares benignos y lesión intraepitelial (48). Sin embargo, esta clasificación y las denominaciones son subjetivas y dependen de la experiencia del observador, dificultando algunas veces establecer un diagnóstico concluyente, lo que conlleva a realizar otros exámenes. Además, no siempre es posible garantizar una adecuada correlación citologíacolposcopia-biopsia, ni una predicción acerca de la evolución futura de una neoplasia. Y como afirma Lacruz: "Ocasionalmente las predicciones se frustran por la notable discrepancia entre el aspecto morfológico de un tumor y su comportamiento biológico" (2). 
Tabla 1. Valores teóricos y prácticos de medidas obtenidas mediante la simulación computacional para el citoplasma (C), núcleo (N) y célula o totalidad (T).

\begin{tabular}{|c|c|c|c|c|c|c|}
\hline \multirow[b]{2}{*}{ DIAGNÓSTICO } & \multicolumn{3}{|c|}{ DIMENSION FRACTAL } & \multicolumn{3}{|c|}{ VARIABILIDAD FRACTAL Y AMI } \\
\hline & $\mathbf{C}$ & $\mathbf{N}$ & $\mathbf{T}$ & C Y N & NYT & C Y T \\
\hline Normal & 1,9245 & 1,4567 & 1,9173 & 5 & 5 & 0 \\
\hline Normal & 1,8764 & 1,2718 & 1,8597 & 6 & 6 & 0 \\
\hline Normal & 1,8341 & 1,4934 & 1,8219 & 4 & 4 & 0 \\
\hline Normal & 1,8563 & 1,3902 & 1,832 & 5 & 5 & 0 \\
\hline Intermedia & 1,7433 & 1,3628 & 1,7247 & 4 & 4 & 0 \\
\hline Intermedia & 1,8432 & 1,329 & 1,8659 & 5 & 5 & 0 \\
\hline Normal & 1,9387 & 1,3976 & 1,9238 & 6 & 6 & 0 \\
\hline Normal & 1,8765 & 1,5293 & 1,8654 & 3 & 3 & 0 \\
\hline ASCUS & 1,5852 & 1,4434 & 1,5537 & 1 & 1 & 0 \\
\hline ASCUS & 1,8078 & 1,3364 & 1,8619 & 5 & 5 & 0 \\
\hline ASCUS & 1,7632 & 1,3769 & 1,6423 & 4 & 3 & 1 \\
\hline ASCUS & 1,5905 & 1,272 & 1,6671 & 3 & 4 & 1 \\
\hline ASCUS & 1,7075 & 1,7331 & 1,7295 & 0 & 0 & 0 \\
\hline ASCUS & 1,866 & 1,4058 & 1,8842 & 4 & 4 & 0 \\
\hline ASCUS & 1,312 & 1,2991 & 1,5481 & $1 e$ & 3 & 2 \\
\hline ASCUS & 1,7568 & 1,3188 & 1,8799 & 4 & 5 & 1 \\
\hline ASCUS & 1,5967 & 1,5685 & 1,5373 & 0 & 0 & 0 \\
\hline ASCUS & 1,7296 & 1,2868 & 1,685 & 5 & 4 & 1 \\
\hline $\mathrm{ASCH}$ & 1,4923 & 1,3461 & 1,4854 & 1 & 1 & 0 \\
\hline $\mathrm{ASCH}$ & 1,1842 & 1,2778 & 1,2196 & 1 & 0 & 1 \\
\hline $\mathrm{ASCH}$ & 1,1933 & 1,2195 & 1,3061 & 1 & 1 & 2 \\
\hline $\mathrm{ASCH}$ & 1,1538 & 1,312 & 1,2209 & 2 & 1 & 1 \\
\hline $\mathrm{ASCH}$ & 1,4291 & 1,2395 & 1,4557 & 2 & 2 & 0 \\
\hline $\mathrm{ASCH}$ & 1,3263 & 1,2184 & 1,3919 & 1 & 1 & 0 \\
\hline L-SIL & 1,5014 & 1,4711 & 1,6857 & 1 & 2 & 1 \\
\hline L-SIL & 1,6867 & 1,4663 & 1,4973 & 2 & 0 & 2 \\
\hline L-SIL & 1,5273 & 1,6474 & 1,5304 & 1 & 1 & 0 \\
\hline L-SIL & 1,5759 & 1,5341 & 1,5273 & 0 & 0 & 0 \\
\hline L-SIL & 1,6407 & 1,4711 & 1,5304 & 2 & 1 & 1 \\
\hline L-SIL & 1,4144 & 1,3979 & 1,5014 & 1 & 2 & 1 \\
\hline L-SIL & 1,5759 & 1,3979 & 1,466 & 2 & 1 & 1 \\
\hline H-SIL & 1,2319 & 1,3188 & 1,256 & 1 & 1 & 0 \\
\hline H-SIL & 1,256 & 1,3157 & 1,343 & 1 & 0 & 1 \\
\hline H-SIL & 1,2252 & 1,2187 & 1,2319 & 0 & 0 & 0 \\
\hline H-SIL & 1,3794 & 1,1609 & 1,1224 & 2 & 0 & 2 \\
\hline H-SIL & 1,8353 & 1,6975 & 1,8353 & 2 & 2 & 0 \\
\hline H-SIL & 1,8013 & 1,6338 & 1,7702 & 2 & 1 & 1 \\
\hline H-SIL & 1,6682 & 1,6727 & 1,669 & 0 & 0 & 0 \\
\hline Carcinoma & 1,7425 & 1,7932 & 1,7481 & 0 & 0 & 0 \\
\hline Carcinoma & 1,6976 & 1,6484 & 1,6114 & 0 & 0 & 0 \\
\hline Carcinoma & 1,2764 & 1,2623 & 1,2298 & 0 & 0 & 0 \\
\hline Carcinoma & 1,3363 & 1,3671 & 1,3954 & 0 & 0 & 0 \\
\hline Carcinoma & 1,6825 & 1,6465 & 1,6789 & 0 & 0 & 0 \\
\hline Carcinoma & 1,4179 & 1,48454 & 1,4985 & 0 & 0 & 0 \\
\hline Carcinoma & 1,3345 & 1,3462 & 1,3185 & 0 & 0 & 0 \\
\hline Carcinoma & 1,7843 & 1,7314 & 1,7752 & 0 & 0 & 0 \\
\hline Carcinoma & 1,4106 & 1,4372 & 1,4292 & 0 & 0 & 0 \\
\hline Carcinoma & 1,3344 & 1,3368 & 1,3083 & 0 & 0 & 0 \\
\hline Carcinoma & 1,6742 & 1,6157 & 1,6197 & 0 & 0 & 0 \\
\hline
\end{tabular}

Las medidas resaltadas en gris corresponden a las medidas de las cinco células ASCUS y las cinco cancerígenas evaluadas empíricamente, evidenciando que las células ASCUS pueden presentar valores correspondientes a estados de normalidad o anormalidad, mientras que las células cancerígenas presentan relaciones de anormalidad con valores de 0 en las tres relaciones. 
El diagnóstico matemático basado en la geometría fractal es una metodología objetiva y reproducible de aplicación clínica, que puede utilizarse para la detección temprana de lesiones cervicales. Se basa en el análisis de la estructura celular, utiliza como herramienta principal la citología, que es un procedimiento no invasivo que facilita la toma de las muestras. La generalización lograda sigue la misma línea que el trabajo en el que se determinó la totalidad de prototipos arteriales en el proceso de estenosis (27), demostrando que el hallazgo de órdenes armónicos entre partes y totalidad de objetos irregulares permite establecer medidas diferenciales entre normalidad y enfermedad, así como una comprensión global del fenómeno, determinando un número finito de prototipos posibles en la práctica.

La AMI y la variabilidad relacionan matemáticamente las partes y la totalidad de la célula, permitiendo establecer una medida matemática que diferencia células normales de anormales, como se evidenció en el diagnóstico desarrollado previamente. La cuantificación de esta relación muestra los diferentes estados celulares, que son casos específicos de prototipos generales que se hallan a partir de permutaciones de estas relaciones. La metodología diagnóstica desarrollada previamente permitió establecer que una célula ASC-US puede tener las mismas relaciones de AMI y variabilidad que una célula normal o clasificada como L-SIL. Al obtener esta generalización se observa que las células ASC-US pueden presentar valores en sus relaciones iguales a los de cualquier prototipo desde la normalidad hasta L-SIL, demostrando desde una sustentación física y matemática, objetiva y reproducible por qué la clasificación ASCUS reúne a células cuyo diagnóstico es indeterminado, y evidenciando por qué estas células confunden a un observador que las evalúa desde una perspectiva subjetiva y cualitativa.

Al comparar los resultados con las mediciones empíricas de células ASCUS se encontró que éstas presentan medidas correspondientes tanto a normalidad como a anormalidad, en tanto que las relaciones halladas para las células cancerígenas presentan los mínimos valores posibles de anormalidad en las relaciones entre $\mathrm{C}$ y $\mathrm{N}$ y entre $\mathrm{N}$ y $\mathrm{T}$.

De igual manera esta metodología permite predecir si las células ASCUS también pueden presentar valores de una célula con cáncer, ayuda a estratificar más específicamente los casos individuales frente a la mirada poblacional y elimina la subjetividad de los métodos actuales. Entonces se puede pensar que tiene tres ventajas: es objetiva, reproducible y además, específica para cada persona y por lo tanto preventiva, pues cuando se encuentran células ASCUS se podrían diferenciar matemáticamente que tan cercanas son a la normalidad, o a los diferentes grados de lesión preneoplásica o a carcinoma, permitiendo así tomar las decisiones clínicas más apropiadas. La aplicación de esta metodología, la cual permitiría clarificar el riesgo real de cada paciente, se realizaría después de que el citólogo realice una primera evaluación con la metodología usual y reporte especialmente células clasificadas como ASCUS, para establecer así matemáticamente los casos en los que es necesario hacer un seguimiento preventivo.

En la era de la mecánica newtoniana, el programa de investigación válido para cualquier área fue el determinismo basado en la causalidad, que fue cuestionado al desarrollarse la mecánica estadística (49), la teoría del caos $(50,51)$, y la teoría cuántica (52). En estas teorías la causalidad dejó de ser fundamento para entender la naturaleza; desde esta perspectiva caótico determinista acausal, Prigogine (53) considera que sólo tenemos ventanas temporales para la evaluación de los fenómenos. Siguiendo esta perspectiva, esta metodología se desarrolló desde un punto de vista donde las relaciones causa efecto no se consideran; es por esto que es independiente de la edad, factores de riesgo y de cualquier análisis poblacional, pues lo que se observa son ventanas temporales de las células, parte de relaciones entre los objetos estudiados y revela un orden armónico subyacente a la irregularidad fractal de la estructura celular, estableciendo el número finito de posibles configuraciones estructurales que abarcan cualquier irregularidad posible del universo. De este modo permite predecir todas las irregularidades fractales de las células cancerígenas y sus relaciones, así como explicar matemáticamente las dificultades diagnósticas de las células ASC-US.

\section{Aplicaciones Futuras}

A partir de medidas realizadas con esta metodología en bancos de datos especializados en lesiones preneoplasicas y cáncer de cuello uterino se confirmaría la aplicabilidad clínica de este método.

\section{Dedicación}

A los niños de Colombia, en especial al ahijado del primer autor Joshua Bahamón Rodríguez. 


\section{Agradecimientos}

Este proyecto es resultado del proyecto MED577, financiado por el Fondo de Investigaciones de la Universidad Militar Nueva Granada. Agradecemos a la Universidad Militar Nueva Granada, especialmente al Dr. José Ricardo Cure Hakim, vicerrector de investigaciones, al Dr. Henry Acuña, Jefe de la división de Investigación Científica y a los doctores Clara Benavides, Sandra Moreno, Germán Forero y Juan Miguel Estrada de la Facultad de Medicina por apoyar las investigaciones físicas y matemáticas aplicadas a la medicina. Agradecemos a las doctoras Constanza Gómez, jefe del laboratorio, y Martha Vega, citotecnóloga, del Laboratorio de Patología del Liga Contra el Cáncer, seccional Bogotá, por proporcionar las muestras necesarias para este trabajo.

\section{Glosario}

Fractal: del latín fractus, interrumpido. Objeto irregular. Término que se define como sustantivo: objeto irregular, y como adjetivo: irregularidad.

Dimensión fractal: medida numérica adimensional del grado de irregularidad de un fractal. La definición de dimensión fractal más usada es la dimensión fractal de box-counting.

Prototipo celular fractal: Combinación simultánea de relaciones numéricas entre las dimensiones fractales de los objetos estudiados: núcleo, citoplasma sin núcleo y totalidad evaluadas con la AMI y Variabilidad.

AMI celular y Variabilidad: Grado de similitud o diferencia entre las dimensiones fractales de dos de los objetos definidos, en este caso se compararán el citoplasma sin núcleo y núcleo ( $\mathrm{C}$ y $\mathrm{N})$, el núcleo y la totalidad ( $\mathrm{N}$ y T) y citoplasma sin núcleo y totalidad $(\mathrm{C}$ y $\mathrm{T})$, ver tabla 1 . Este valor se calcula restando la primera cifra significativa de las dimensiones fractales de los dos objetos que se van a comparar; el valor absoluto de la resta realizada es el valor de AMI celular y Variabilidad.

\section{Referencias}

1. Robbins S. Aparato genital femenino. En: Robins S, editores. Patología Estructural y Funcional. $7^{\circ}$ ed. Madrid: McGraw-Hill Interamericana de España S.A., 1996; p. 1156-1160.

2. Lacruz C. Nomenclatura de las lesiones Cervicales (de Papanicolau a Bethesda 2001). Rev Esp Patol. 2003;36(1):1-5.

3. Rodríguez J, Álvarez L, Mariño M, Avilán G, Prieto S, Casadiego E, Correa C, Osorio E. Variabilidad de la Dimensión Fractal del Árbol Coronario Izquierdo en Pacientes con Enfermedad Arterial Oclusiva Severa. Dinámica Fractal de la Ramificación Coronaria. Rev Col Cardiol. 2004;11(4):185-92.

4. Rodríguez J, Prieto S, Ortíz L, Correa C, Álvarez L, Bernal P, Casadiego E. Variabilidad de la Dimensión Fractal de la Ramificación Coronaria Izquierda en Ausencia y Presencia de Enfermedad Arterial Oclusiva Moderada y Severa. Rev Col Cardiol. 2007;14(3):173-79.

5. Rodríguez J, Mariño M, Avilán N, Echeverri D. Medidas Fractales de Arterias Coronarias, un Modelo Experimental en Reestenosis, Armonía Matemática Intrínseca de la Estructura Arterial. Rev Col Cardiol. 2002; 10:65-72.

6. Mandelbrot B. Introducción. En: Mandelbrot B. Los Objetos Fractales. Barcelona, España: Tusquets Eds. S.A., 1998; p.13-26.
7. Mandelbrot B. The Fractal Geometry of Nature. San Francisco: Freeman; 1972.

8. Peitgen H. Length, Area and Dimension. Measuring Complexity and Scalling Properties. En: Peitgen H, Jürgens H, Saupe D. Chaos and Fractals: New Frontiers of Science. New York: Springer-Verlag; 1992; p.183-228.

9. Goldberger Al. Fractal Dynamics in Physiology: Alterations with Disease and Aging. Proceeding of the National Academy of Sciences. 2002;99:2466-2472.

10. Goldberger A, Rigney D, West B. Chaos and Fractals in Human Physiology. Sci Am. 1990;262:42-49.

11. Kiselev V G, Hahn K R, Auer D P. Is the Brain Cortex a Fractal? Neuroimage. 2003;20(3):1765-74.

12. Cheng S C, Huang Y M. A Novel Approach to Diagnose Diabetes Based on the Fractal Characteristics of Retinal Images. IEEE Trans Inf Technol Biomed. 2003;7(3):163-70.

13. Koshino T, Kimura Y, Kameyama Y, Takahashi T, Yasui T, Chisaka H, et al. Fractal and Periodic Heart Rate Dynamics in Fetal Sheep: Comparison of Conventional and New Measures Based on Fractal Analysis. Am J Physiol Heart Circ Physiol. 2003;284(5):1858-64.

14. Mauroy B, Filoche M, Weibel ER, Sapoval B. An optimal bronchial tree may be dangerous. Nature 2004;427:633-636.

15. Garte S. Fractal properties of the human genome. J Theor Biol 2004;230(2):251-60.

16. West BJ. Fractal Physiology and Chaos Medicine. London: World Scientific Publishing Co; 1990.

17. Baish HJ, Jain R. Fractals and Cancer. Cancer Research. 2000;60:3683-88.

18. Dey P, Rajesh L. Fractal Dimension in Endometrial Carcinoma. Anal Quant Cytol Histol. 2004;26(2):113-6.

19. Kikuchi A, Kozuma S, Yasugi T, Taketani Y. Fractal Analysis of the Surface Growth Patterns in Endometrioid Endometrial Adenocarcinoma. Gynecol Obstet Invest. 2004;58(2):61-7.

20. Gazit Y, Berk D A, Leunig M. Scale-invariant Behavior and Vascular Network Formation in Normal and Tumor Tissue. Phys Rev Lett. 1995;75:2428-31.

21. Gazit Y, Baish JW, Safabaksh N. Fractal Characteristics of Tumor Vascular Architecture during Tumor Growth and Regression. Microcirculation. 1997;4(4):395-402.

22. Pohlman S, Powell K, Obuchowski N A. Quantitative Classification of Breast Tumors in Digitized Mammograms. Med Phys. 1996;23:1337-45.

23. Lefebvre F, Benali H. A Fractal Approach to the Segmentation of Microcalcifications in Digital Mammograms. Med Phys. 1995;22:381-90.

24. Landini G, Rippin J W. Fractal Dimensions of Epithelialconnective Tissue Interfaces in Premalignant and Malignant Ephitelial Lesions of the Floor of Mouth. Anal Quant Cytol Histol. 1993;15:144-49.

25. Luzi P, Bianciardi G. Fractal Analysis in Human Pathology. Ann NY Acad Sciences. 1999;879:255-57.

26. Rodríguez J, Prieto S, Ortiz L, Wiesner C, Díaz M, Correa C. Descripción Matemática con Dimensiones Fractales de Células Normales y con Anormalidades Citológicas de Cuello Uterino. Rev Cienc Salud. 2006;4(2):58-63.

27. Rodríguez J, Prieto S, Correa C, Bernal P. Theoretical Generalization of Normal and Sick Coronary Arteries with Fractal Dimensions and the Arterial Intrinsic Mathematical Harmony. BMC Medical Physics. 2010;10:1. http://www.biomedcentral. com/1756-6649/10/1.

28. Rodríguez J, Prieto S, Ortiz L, Avilán N, Álvarez L, Correa C, Prieto I. Comportamiento Fractal del Ventrículo Izquierdo Durante la Dinámica Cardíaca. Rev Colomb Cardiol. 2006;13(3):165-70. 
29. Rodríguez J, Prieto S, Ortiz L, Ronderos M, Correa C. Diagnóstico Matemático de Ecocardiografías Pediátricas con Medidas de Dimensión Fractal Evaluadas con Armonía Matemática Intrínseca. Rev Colomb Cardiol. 2010;17(2):79-86.

30. Rodríguez J, Prieto S, Ortiz L, Bautista A. Diagnóstico Matemático de la Monitoria Fetal Aplicando la Ley de Zipf-Mandelbrot. Rev Fac Med Univ Nac Colomb. 2006;54(2):96-107.

31. Rodríguez J. Dynamical Systems Theory and ZIPF - Mandelbrot Law Applied to the Development of a Fetal Monitoring Diagnostic Methodology. Proceedings of the XVIII FIGO World Congress Of Gynecology And Obstetric. November 2006, Kuala Lumpur, Malaysia.

32. Rodríguez J, Correa C, Prieto S, Ospino B, Bernal P, Ortiz L, Munévar A. Caracterización Geométrica de los Glóbulos Rojos. Diferenciación de Muestras Normales y Patológicas. Rev Cienc Salud. 2008;6(3):47-56

33. Rodríguez J. Diferenciación Matemática de Péptidos de Alta Unión de MSP-1 Mediante la Aplicación de la Teoría de Conjuntos. Inmunología. 2008;27(2):63-68.

34. Rodríguez J. Caracterización Física y Matemática de Péptidos de Alta Unión de MSP-1 Mediante la Aplicación de la Teoría de la Probabilidad y la Entropía. Archivos de alergia e inmunología clínica. 2008;39(2):74-82.

35. Rodríguez J, Correa C, Prieto S, Puerta G. Aplicación de la Probabilidad y la Entropía a la Proteína EBA-140. Caracterización Matemática de Péptidos de Alta Unión. Inmunología. 2009;28(2):65-73.

36. Rodríguez J, Correa C, Prieto S, Cardona D, Vitery S, Puerta G, Soracipa Y, Bernal P. Caracterización Física y Matemática de Péptidos de Alta Unión de MSA-2: Aplicación de la Teoría de la Probabilidad y la Entropía. Rev Acad Colomb Cienc. 2009;33(129):549-557.

37. Rodríguez J. Teoría de Conjuntos Aplicada a la Caracterización Matemática de Unión de Péptidos al HLA clase II. Rev Cienc Salud. 2008;1:9-15

38. Rodríguez J, Bernal P, Correa C, Prieto S, Benítez L, Viteri S, et al. Predicción de Unión de Péptidos de MSA-2 y AMA-1 de Plasmodium falciparum al HLA clase II. Inmunología. 2009;28(3):115-24.

39. Rodríguez J, Bernal P, Álvarez L, Pabón S, Ibáñez S, Chapuel $\mathrm{N}$, et al. Predicción de Union de Péptidos de MSP-1 Y EBA140 de Plasmodium Falciparum al HLA Clase II: Probabilidad, Combinatoria y Entropía Aplicadas a Secuencias Peptídicas. Inmunología. 2010;29(1):7-19.
40. Rodríguez J. Teoría de unión al HLA clase II:Teoría de Probabilidad, Combinatoria y Entropía Aplicadas a Secuencias Peptídicas. Inmunología. 2008;27(4):151-166.

41. Rodríguez J, Bernal P, Prieto S, Correa C . Teoría de Péptidos de Alta Unión de Malaria al Glóbulo Rojo. Predicciones Teóricas de Nuevos Péptidos de Unión y Mutaciones Teóricas Predictivas de Aminoácidos Críticos. Inmunología. 2010;29(1):7-19.

42. Rodríguez J, Prieto S, Avilán N, Correa C, Bernal P, Ortiz L, et al. Nueva Metodología Física y Matemática de Evaluación del Holter. Rev Colomb Cardiol. 2008;15:50-54.

43. Rodríguez J, Correa C, Ortiz L, Prieto S, Bernal P, Ayala J. Evaluación Matemática de la Dinámica Cardíaca con la Teoría de la Probabilidad. Rev Mex Cardiol. 2009;20(4):183-9.

44. Rodríguez J. Entropía Proporcional de los Sistemas Dinámicos Cardiacos: Predicciones Físicas y Matemáticas de la Dinámica Cardíaca de Aplicación Clínica. Rev Colomb Cardiol. 2010;17:115-29.

45. Rodríguez J. Dinámica Probabilista Temporal De La Epidemia De Malaria En Colombia. Rev Fac Med 2009;17(2):214-21.

46. Rodríguez J, Correa C. Predicción Temporal de la Epidemia de Dengue en Colombia: Dinámica Probabilista de la Epidemia. Revista Salud Pública. 2009;11(3):443-53.

47. Rodríguez J. Método para la Predicción de la Dinámica Temporal de la Malaria en los Municipios de Colombia. Rev Panam Salud Publica. 2010;27(3):211-8.

48. De Vita V Jr.,Hellman S, Rosenberg S. Principios y Práctica de Oncología. 5 a ed. Bogotá, Colombia: Editorial Medica Panamericana, ARAN ediciones S.A.; 1997.

49. Feynman R. Los Principios de la Mecánica Estadística. En: Feynman R. Física. Vol. 1. Cap. 40. Wilmington: Addison-Wesley Iberoamericana S.A., 1987; p. 40-1 - 40-15.

50. Fernandez - Rañada A. Movimiento Caótico. En: Rañada A. Orden y Caos. Scientific American. Prensa Científica S.A., 1990; p. 66-77.

51. Crutchfield J, Farmer D, Packard N, Shaw R. Caos. En: Rañada A. Orden y Caos. Scientific American. Prensa Científica S.A., 1990; p. 78-90.

52. Feynman R. Leighton RB, Sands M. Comportamiento Cuántico. En: Feynman R. Leighton R B, Sands M. Física Vol 1, Cap 37. Wilmington: Addison-Wesley Iberoamericana S.A., 1987; p. 37-1 - 37-16.

53. Fernandez - Rañada A. Introducción. En: Rañada A. Orden y Caos. Scientific American. Prensa Científica S.A., 1990; p. 4-8. 